\title{
Tradeoffs in Design-to-Budget Projects
}

\author{
Franck M. Chatel ${ }^{1}$ and Wilfried Kruse ${ }^{2}$ \\ DLR, Oberpfaffenhofen, Wessling, 82234, Germany \\ Ralf Faller ${ }^{3}$ \\ DLR, Oberpfaffenhofen, Wessling, 82234, Germany \\ and \\ Michael Schmidhuber ${ }^{4}$ \\ DLR, Oberpfaffenhofen, Wessling, 82234, Germany
}

\begin{abstract}
This paper aims at investigating a method to cope with tradeoffs in Design-to-Budget projects. It focuses only on mission operations and does not consider aspects related to spacecraft design, launcher or insurance.
\end{abstract}

The possible tradeoffs depend upon different criteria (e.g. system availability, operational risk), whose relative importance is in turn imposed by the mission characteristics (e.g. recurring satellite routine operations, human spaceflight, LEOP). A methodology, issued from the optimization field, is first defined in order to evaluate the impact of these tradeoffs on the considered criteria. The methodology allows taking the mission characteristics into account and the allocated budget is modeled as a constraint of the problem. A list of cost reduction measures is then produced, supported by GSOC experience or the existing literature, together with an assessment of their impact on the criteria. Finally the methodology is applied in relation with a budget level to find out which cost reduction measures would satisfy the budget constraint and which criteria would be impacted.

\section{Introduction}

his paper summarizes some considerations made at the German Space Operations Centre (GSOC) about

tradeoffs in Design-to-Budget missions. These considerations were prompted when discussing contractual issues with a customer. The total price of the submitted proposal exceeded what the customer was willing to pay for. To meet the customer's requested price, it was necessary to reduce the foreseen service level. An implicit constraint from both GSOC management and the customer was that this reduction of service level shall almost not impact the overall quality of the service delivered.

This is a typical design-to-budget situation. Tradeoffs on the delivered service items must be made to comply with the cost constraint. No expertise about decision-making methods was available at GSOC and the proposed method is the very first in the field.

\section{Methodology}

The following sections details the method used to cope with the problem.

\footnotetext{
${ }^{1}$ Flight Operations Manager, Mission Operations, Franck.Chatel@dlr.de.

${ }^{2}$ Ground Data System Manager, Communication and Ground Stations, Wilfried.Kruse@dlr.de.

${ }^{3}$ Project Manager, Mission Operations, Ralf.Faller@dlr.de.

${ }^{4}$ Training Manager, Mission Operations, Michael.Schmidhuber@dlr.de.
} 


\section{A. Formulation of the problem}

The proposal provided to the customer contains a description of the service, including the products to be delivered, and a cost estimation. The search for tradeoffs is usually performed within cost-benefit analysis and trade studies, which are related to the decision analysis and operational research disciplines. As a matter of fact, tradeoffs are really a matter of making a decision in front of different alternatives. Many methods were developed for decision analysis, like, for example, the Multi-Attribute Utility Theory (MAUT) and the Analytic Hierarchy Process (AHP).

In the spirit of the MAUT method ${ }^{1,2,3}$, the problem was reformulated as a design and optimization problem: find out which products shall be offered so that the quality of the service is maximized under the constraint that the overall cost remains under a given limit. This is first a design problem in the sense that one searches for a combination of the products to offer. This is similar to choosing the sensors and actuators when designing a spacecraft: tradeoffs shall be made between cost, performance and robustness. The design-to-budget nature of the problem appears clearly as a constraint whereas maximizing the "quality of service" reflects the aim of delivering the best service possible.

As in all optimization problems, it is necessary to quantify the cost of each service or product. This can be done based on experience and data from previous missions (if possible comparable). Measuring the "quality of service" is more difficult: some criteria (also called attributes or objectives), such as flexibility or availability for example, shall be defined for the assessment. At this point, the concept of utility functions is introduced to evaluate how much an alternative contributes to each criterion. The shape of the utility function is closely related to the risk attitude of the decision maker. Due to the lack of expertise about utility functions, it has been decided to rely on a well-document method and use AHP to compute a multi-linear utility function.

\section{B. Tree of alternatives and cost estimation}

First, the term "alternative" shall be defined more precisely. In typical decision-making problem, the alternatives represent different products (cars for example) that are mutually exclusive and the decision-maker wants to choose between these alternatives. Translated to the present case, an alternative would represent all combinations of services and products listed in the proposal, including the different levels of workload associated. For example conducting flight operations can be done with three full shifts of eight hours or one full shift of ten hours and two reduced shifts of seven hours. On the other hand flight procedures might be developed from scratch or slightly customized from the inputs delivered by the customer. The service "flight operations" and the product "flight operations procedures" with their aforementioned workload yield already four alternatives!

The previous example shows that the number of alternatives would be very high for a real case. The different workload levels associated to a service or product are exactly the variables to adjust and they relate directly to the cost of the service or product. To be able to make tradeoffs, it is interesting to have as many such variables as possible, which drives the number of alternatives to unrealistic high values. This situation arises because the service and products are not structured, which implies to consider all combinations.

At this stage, the $\mathrm{AHP}^{4}$ approach has been used to introduce a hierarchy between the different services or products and their associated workload. The goal and criteria, that is the first two levels of the hierarchy, will be defined in the next section. The third level of the hierarchy lists all the services and products listed by the customer in the Request for Proposal (RfP). The fourth level lists the different tasks involved in delivering the service or product. For example writing Flight Operations Procedures (FOP) requires importing them in a tool, developing them and finally validating them. Finally the fifth and last level corresponds to the workload levels. An obvious workload level, applicable to all services and products, is simply to waive the service or product: the associated cost is then null and nothing will be delivered. Proposing different workload levels for a service or product and estimating the associated cost is a central task in solving the problem. Taking the example of the task related to FOP validation, the usual workload level is to validate procedures using a delivered software simulator. Other workload levels could include a review by satellite manufacturer experts or a validation

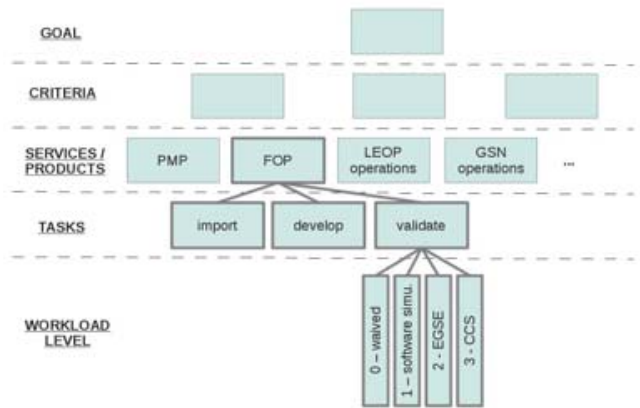

Figure 1: Example of hierarchy structure directly with the Electrical Ground Support Equipment (EGSE, e.g. in case no software simulator is available. This conflicts with integration tasks however and often requires working on weekend). Another possibility would be to set-up a Central Checkout System (CCS) used commonly for Assembly, Integration and Tests purposes by the satellite manufacturer and by the operations team to validate the FOPs: this implies more work 
to set-up but FOP development would benefit from integration tests. An example of hierarchy structure can be seen on Figure 1.

The introduction of the three first hierarchical levels structures the problem. The fourth and fifth levels correspond to the tasks and workload levels associated to the parent node. Both items are specific to the parent and, as such, irrelevant for the other nodes. This allows restricting the comparisons of the tasks and workload levels to the parent node, which actually reduces the number of comparisons to be performed.

\section{Criteria of quality and utility functions}

The first and second levels of the hierarchy introduced at the previous section correspond to the goal and the criteria to measure the quality. The goal is defined as the mission success. It could be surprising to formulate the goal like this, instead of using a more natural formulation like fulfilling the contract. This is done on purpose because the experience of the GSOC decision maker is implicitly required as opposed to fulfilling contractual obligations without thinking about the mission.

In the frame of spacecraft operations, following criteria were considered as relevant:

- quality of ground processes

- availability of ground system

- safety of operations

- risk for project achievement

Of course additional criteria might be considered depending on the type of mission. For example the security of the operations is relevant for military missions or the ergonomics of the ground system in case of robotic missions. This remark highlights that the mission context, as defined by the customer in the RfP, shall be taken into account at this stage. The mission context, although not explicitly mentioned, conditions the criteria of quality as well as the comparisons between alternatives. In case some details remain unknown, such as the number of flight procedures to develop or the complexity of the spacecraft, some assumption must be made and documented. Basically, the mission context affects the first three levels (overall goal, quality criteria and services/products) of the hierarchy whereas the last two levels (tasks and workload levels) reflect the work organization.

Having chosen the criteria, the next step is to define how the utility function associated to an alternative is computed. Utility ${ }^{5}$ functions are issued from the economics and measure the satisfaction a customer finds in a purchased good. Transposed to the present problem, utility functions characterize the contribution of a service or product to the overall goal. Quite some literature is available on utility functions. Due to the use of a hierarchy as prescribed by the AHP method, it is convenient to introduce weights to characterize the relative importance of the children nodes for their parent node. These weights are functionally equivalent to the AHP local priorities but can be different, thus allowing remaining independent of the AHP method. The utility value of a workload level with respect to a quality criterion was defined as being the product of the weights of the branch (i.e. the path between the criterion node and the leaf representing the workload level). An alternative being the combination of all workload levels, the utility value of an alternative with respect to a criterion is further defined as the sum of the utility values of all involved workload

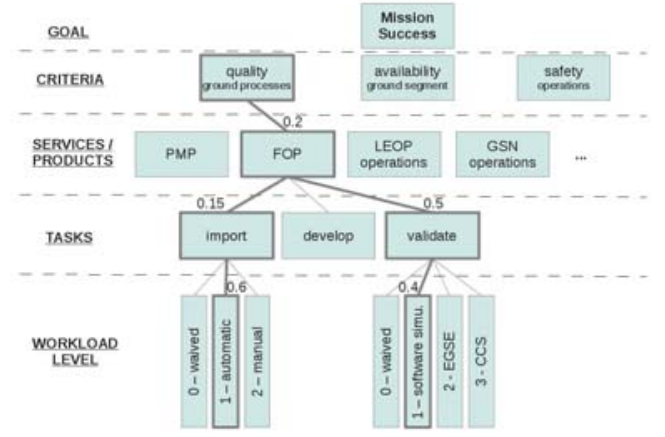

Figure 2: Hierarchy structure with weights levels with respect to the same criterion.

Based on the example given in figure 2, one can compute:

- Utility value of validating with software simulator with respect to quality criterion is 0.04 (product of weights $0.2,0.5$ and 0.4 ).

- Utility value of importing FOP automatically with respect to quality criterion is 0.018 .

- Utility value of highlighted alternative with respect to quality criterion is $0.04+0.018$.

The proposed model is basically linear in all variables (i.e. the work levels), which means that possible interactions between alternatives are not taken into account. This is strictly speaking not true: for example the more complex the design of the ground segment, the more training is needed to achieve the same level of proficiency. It was considered as an acceptable simplification at this stage. However the pertinence of such a model will be questioned after first exercising the methodology. 


\section{Putting all pieces together}

At this point, a hierarchy has been developed and cost estimate attached to each leaf of the tree. Consequently, the way of setting the weights shall now be defined. Within the MAUT ${ }^{1,2,3}$ methodology, these weights can be specified as ratio or by solving a system reflecting the tradeoffs between objectives. Due to its simplicity, it was decided to apply the AHP process and identify the weights with the local priorities. This choice presents the advantage of a clear methodology ${ }^{4}$.

Once the weights have been computed, the last step consists in solving the optimization problem. Let us note $N$ the total number of tasks and $q_{j}$ the number of workload levels of task $j(j \leq N)$. Let us further introduce $\varepsilon_{j k}$ a boolean corresponding to the $k^{\text {th }}$ workload level of task $j\left(j \leq N\right.$ and $\left.k \leq q_{j}\right)$. In order to formalize the problem, one defines the vector $A=\left(\varepsilon_{11} \ldots \varepsilon_{1 q 1} \varepsilon_{21} \ldots \varepsilon_{2 q 2} \ldots \varepsilon_{N 1} \ldots \varepsilon_{N q N}\right)^{t}$ representing an alternative and $U_{p}(A)$ its utility value with respect to the $p^{\text {th }}$ criterion ( $p \leq P$, where $P$ is the total number of criteria). Given the definition of the utility function in the previous section, one can write $U_{p}(A)=M . A$, where $M$ is a matrix containing all the weights derived of the AHP pairwise comparisons. The cost of an alternative is simply the sum of the costs of all involved workload levels, which is simply expressed by $C(A)=C \cdot A, C$ being a vector containing the costs of each workload level. Finally it is necessary to add $N$ constraints stating that one and only one workload level shall be defined for a given task, which can be simply formulated as $\varepsilon_{j l}+\varepsilon_{j 2}+\ldots+\varepsilon_{j q j}=1$ for each $j$. This can be summarized as a linear system of constraints $D . A=1_{N}$, whith $l_{N}$ being a vector of size $\mathrm{N}$ whose components are all equal to 1 . With these notations, the problem can be written as:

$$
\max _{A}\left[U_{I}(A), U_{2}(A), \ldots, U_{P}(A)\right] \text { subject to the constraints } C(A) \leq C_{\max } \text { and } D . A=1_{N}
$$

This is a typical Binary Integer Linear Programming problem, a particular case of Multiple Objective Integer Programming 8 . Its resolution usually consists in finding the set of non-dominated (so-called Pareto optimal) points. Such a problem is difficult (NP-hard) from the computational point of view. In the present case, it was chosen to aggregate the utility functions into a single objective function (weighted sum with weights computed by AHP method) and apply a dedicated solver (lp_solve).

\section{Results}

The methodology described previously has been applied to a real project but unfortunately too late to support the decision about the tradeoffs. The starting point was the proposal delivered to the customer and the related cost estimation. First a hierarchy for the services/products (this is an additional layer with respect to the previous section) has been developed from the work package structure attached to the proposal. Using this package structure as basis for the hierarchy is quite natural but only the first level, comprising nine packages, has been used. Services and products were grouped into forty larger pools, allowing a relatively fine granularity without driving the complexity too high. Only one task has been defined as far as possible for a service or product, again to keep the complexity under control. However it was necessary to define more than one task when commonalities were not provided within the pool or when the cost structure made it useful. The possible tradeoffs and their associated cost estimation have been defined at the workload level, mostly based on GSOC experience. The resulting size of the vector of alternatives was hundred twenty seven.

After defining the hierarchy, approximately four hundred pairwise comparisons had to be performed! Experienced engineers in flight operations were chosen for this task, each of them having different focus (project management, software development, ground stations, training). The original plan was to perform the comparison in group to reach a consensus. It quickly resulted in endless discussions! It was decided that each participant would perform the comparisons on his/her own. This situation seems to be common and procedures ${ }^{7}$ have already been developed to aggregate results and check their consistency. All participants were treated equally and weighted identically in the computations. Using a weighted geometric mean ${ }^{6,7}$ to derive the weights and an acceptable consistency ratio ${ }^{7}$ of 0.1 , it turned out that the majority of the AHP matrices were declared consistent. It would have been necessary to discuss inconsistencies case by case. Unfortunately time was short and results have not been corrected (it must be noted that most of the inconsistencies were closed to the limit of $0.1)$.

The computations were performed with the help of Scilab ${ }^{10}$, using lp_solve ${ }^{9}$ to tackle the integer linear programming. Approximately seven hundred fifty lines of code were necessary for all computations and the solution (including reporting) was available within less than a second (CPU: Intel Core Duo, $2.40 \mathrm{GHz}, \mathrm{RAM}$ : $3.9 \mathrm{~GB})$.

In order to assess the solution found by the program, each participant was further asked to propose a solution based on his/her experience. The allowed budget was set around $75 \%$ of the price contained in the proposal, which was already considered as low. It is interesting to note that no participant could find a solution satisfying the cost constraint: proposed solutions were $15 \%$ above the budget limit. Further cost reduction would have 
been unconceivable for them, which shows that the allowed budget was such that the contract was not acceptable. The computed solution, meeting the budget constraint, featured some striking features like leaving flight procedures development and validation out as well as team training! This point will be addressed in the next section.

Finally the proposal manager and the foreseen manager of the project used as example were asked which of the solution would be preferred. It was clearly stated that only one proposition met the cost constraint and that colleagues had not been able to. Both of them chose the cheapest of the participant proposal. When confronted with the fact that the solution did not meet the budget and asked if they would change their mind, both of them deemed the computed solution unacceptable. This confirms that the expected budget was probably too low. Of course, such a result is biased because the computed solution is not comparable in price with the other ones. In order to avoid this bias, a new solution has been computed with a budget comparable (actually the average value) to the one of the participants. Moreover different proposal and project managers (but involved in similar types of mission) were chosen to ensure no prior knowledge of the solution. Both of them chose the computed solution! This is an argument in favor the fact that the previous solution was rejected rather because of its low budget than because of a wrong model.

\section{Lessons learned when using the methodology}

This section is divided into direct comments about the method and possible improvements.

\section{A. Feedback}

The first feedback was that the pairwise comparison process is demanding. This process is not only time consuming, it requires a lot of concentration. This questions the quality of the judgments towards the end of the process, which corresponds to lower portion of the hierarchy (comparisons between workload levels). De facto, all matrices reported as inconsistent by the consistency check were related to the workload levels (performed at the end of the comparison process). The fact that the comparisons of workload levels are linked to the practical daily work, as opposed to those between work packages, reinforces the concern. This is of course detrimental to the entire method because the choice of alternatives is based exactly on the elements where the judgment is questionable. It can only be recommended to discuss the detected inconsistencies, even if it means investing more time in the comparison process. A special display, presenting only the alternatives to be compared and hiding the rest, could also help. However this point clearly limits the complexity of the model that can be analyzed by this method.

A second comment is related to the interpretation of the proposed alternatives, which simply differs with the person, and spoils the pairwise comparison process. It results into incorrect values of utility functions, which could in turn partly explain the apparent contradictions observed in the first optimal solution. As a matter of facts, the value of the utility functions related to important activities such as development or validation of flight procedures was lower than less important tasks. Although these products got higher weights at lower levels, the weight of their parent was low and yielded low global weights. After looking at the results, participants agreed that it did not correspond to their understanding when performing the pairwise comparisons. This is especially the case between the interpretation of safety and risk criteria. With the benefit of hindsight, this probably fueled the endless discussions before it was decided to perform the pairwise comparisons individually. The hierarchy structure should be developed with all the participants to improve the understanding, leaving then open the possibility to compare the items individually or in group.

Finally the granularity introduced when defining the workloads was ill adapted for the process. Some cases, especially documents, were overly detailed whereas other products were taken as a whole without much possible workload levels. This is problematic in two ways: first the comparison process gets confused by subtle differences, thus requiring more concentration. It was already noted that the required concentration was a bottleneck of the method. Moreover activities whose associated workload level where not refined enough, as it was the case for the development and validation of flight procedures or training activities, tend to have a higher cost. Due to the cost constraint that must be satisfied, the method is quite sensitive to such expensive blocks of activities. When the cost is not compensated by high values of the utility functions, these blocks of activities are easily ruled out during the optimization.

The present article is a very first investigation in decision-making methods. This lack of experience in the field explains the difficulties described previously. The authors would welcome any comment or suggestion to improve the methodology.

Besides these main feedbacks, some interesting features shall be pointed out. The proposed method is automatically documented by the pairwise comparison process. It is afterwards easy to interpret the computed 
results. It can even be improved when each participant explains the reasons behind his/her choices but this requires more time and probably using specialized software.

Additional or partial judgments can easily be taken into account by simply computing the affected matrices without touching the rest. The model can be seen as a way of aggregating the knowledge of experts. The fact that the model is separated from the cost estimation allows re-using the results for similar missions where the costs might differ, for example due to the implementation of new tools or standards. This feature was used when computing the second optimal solution. However it must be kept in mind that the mission characteristics are implicitly taken into account in the pairwise comparison process. Consequently one should be careful when applying a model to different mission context.

\section{B. Potential Improvements}

The hierarchy organization treats branches independently. Although this simplification yields an easy-tosolve linear model of the project, it fails capturing interactions. For example investing in tools to automate integration tests of the ground segment might also benefit to the validation of flight procedures. Some simple interactions, such as "if workload level A is chosen, then workload level B is defined", can be modeled as linear constraints but this is limited. This can be improved by resorting to more powerful models such as the Analytic Network Process ${ }^{4}$ (ANP) for example. Another possibility is to increase the complexity of the utility functions by considering products of terms to reflect interactions but the problem will not be linear anymore. One should be careful when increasing the complexity of the model: although computer power increases steadily, integer programming belongs to the class of computationally hard problems even when the model is linear. A way around integer values could be using the concept of fuzzy logic but the interpretation of the computed solution would be made more difficult.

The previous aspect was about interactions within the modeled project. There might also be interactions outside the project: standardization of software, new norms or long-term strategy. Such interactions clearly cannot be taken into account with the proposed method. One possibility could be to define a criterion to measure how well an alternative fits with project external factors.

Estimating the cost related to an alternative is a key activity. Since the cost is taken into account as a constraint to be satisfied, the method is quite sensitive to this estimation. Of course, experience gained in past missions supports this task but often (and hopefully!) the implementation of new technologies shall be considered. Theory ${ }^{3}$ provides means to cope with such issues by using probabilities. Such refinements were not considered at the present stage of investigations.

In order to solve the problem, utility functions were aggregated into a single objective function. It would be interesting to compute the set of non-dominated alternatives instead. This would offer more diversity in the solution but adds the complication to analyze all the solutions and find the preferred one.

\section{Conclusion}

This paper presents a first approach to deal with tradeoffs in design-to-budget missions. A methodology has been developed and applied to a real case. It allows tackling the problem but is time consuming. As such it might be suitable to small and middle-sized projects.

The computed solution had some striking features that were deemed unacceptable by the managers in charge of the project. Since it was the very first time such methods were used, a lack of experience in the implementation of the AHP and MAUT methods have surely induced an inaccurate model. On top of this, the budget of the project chosen for the investigation was probably unrealistic. A second solution having a comparable budget have been computed and gained the favor of the managers this time.

The present investigation is too short to really advocate for or against the proposed methodology. However the second example shows that it has potential for further developments and could possibly support the acquisition process actively. 


\section{Appendix A \\ Acronym List}

$\begin{array}{ll}\text { CCS } & \text { Central Checkout System } \\ \text { FOP } & \text { Flight Operations Procedure } \\ \text { EGSE } & \text { Electrical Ground Support Equipment } \\ \text { GSOC } & \text { German Space Operations Center } \\ \text { MOIP } & \text { Multiple Objective Integer Programming } \\ \text { RfP } & \text { Request for Proposal }\end{array}$

\section{Appendix B \\ Glossary}

AHP

ANP

Analytic Hierarchy Process. Decision-making method based on the development of a tree-like hierarchy to structure the decision process. Evaluation of the hierarchy nodes is made with pairwise comparisons allowing deriving priorities.

Analytic Network Process. Decision-making method generalizing the AHP. The problem is structured as a network instead of a tree.

MAUT Multi-Attribute Utility Theory. Method using utility functions to rank alternatives. MAUT is one of the methods used in multi-criteria decision analysis.

\section{Acknowledgments}

The authors want to thank Armin Braun for his comments and support throughout the investigations.

\section{References}

${ }^{1}$ Olson, D. L., Moshkovich, H. M., Schellenberger and R., Mechitov, A. L., "Consistency and Accuracy in Decision Aids: Experiments with Four Multiattribute Systems”, Decision Sciences, Vol. 26, No. 6, Nov./Dec. 1995.

${ }^{2}$ Henriksen A. D., "Two Decision Analysis Approaches: Choosing a Chemical Analysis Method", Remediation Journal, Vol. 7, No. 3, 2006.

${ }^{3}$ Hannan E. L., Smith J. A. and Gilbert G. R., "A multiattribute decision-making approach to the selection of an auxiliary device for icebreakers", Decision Sciences, Vol. 14, No. 2, 2007.

${ }^{4}$ Saaty, T. L., "The Analytic Hierarchy and Analytic Network Measurement Processes: Applications to Decisions under Risk", European Journal of Pure and Applied Mathematics, Vol. 1, No. 1, 2008, pp.122-196.

${ }^{5}$ Gerber, H. U., and Pafumi, G., "Utility functions: from risk theory to finance", North American Actuarial Journal, Vol. 2, No. 3, pp. 74-91.

${ }^{6}$ Barzilai, J., and Golany, B., "Deriving weights from pairwise comparison matrices: the additive case", Operations Research Letters, 9, 1990, pp. 407-410.

${ }^{7} \mathrm{Xu}, \mathrm{Z}$., "On consistency of the weighted geometric mean complex judgment matrix in AHP", European Journal of Operational Research, 126, 2000, pp.683-687.

${ }^{8}$ Ehrgott, M., and Gandibleux, X., "An Annotated Bibliography of Multiobjective Combinatorial Optimization”, Report in Wirtschaftsmathematik, Nr 62/2000, Apr. 2000.

${ }^{9} \mathrm{lp} \_$solve, Mixed Linear Programming solver, Ver. 5.5.2.0, http://lpsolve.sourceforge.net/

${ }^{10} \mathrm{Scilab}$, software for numerical computation, Ver. 5.3.3, http://www.scilab.org 